\title{
Relationship between Spontaneous Speech Function and Behavior Rating Inventory of Executive Function Profile in Children with Autism Spectrum Disorders: A Pilot Case Study
}

\author{
Kouji Oishi1 ${ }^{*}$, Kunihiko Suto ${ }^{2}$, Asami Nakauchi ${ }^{3}$, Takatsugu Watanabe ${ }^{4}$, \\ Ami Takemori ${ }^{5}$, Maki Toyota ${ }^{1}$ \\ ${ }^{1}$ College of Contemporary Psychology, Rikkyo University, Niiza, Japan \\ ${ }^{2}$ Faculty of Education, Yamaguchi University, Yamaguchi, Japan \\ ${ }^{3}$ Department of Early Childhood Education and Nursing, Seibi Gakuen College, Tokyo, Japan \\ ${ }^{4}$ Faculty of Social Welfare, Rissho University, Kumagaya, Japan \\ ${ }^{5}$ Tokorozawa City Education Center, Tokorozawa, Japan \\ Email: *oishi@rikkyo.ac.jp
}

How to cite this paper: Oishi, K., Suto, K., Nakauchi, A., Watanabe, T., Takemori, A., \& Toyota, M. (2017). Relationship between Spontaneous Speech Function and Behavior Rating Inventory of Executive Function Profile in Children with Autism Spectrum Disorders: A Pilot Case Study. Psychology, 8, 2138-2145.

https://doi.org/10.4236/psych.2017.813136

Received: October 11, 2017

Accepted: November 19, 2017

Published: November 22, 2017

Copyright $\odot 2017$ by authors and Scientific Research Publishing Inc. This work is licensed under the Creative Commons Attribution International License (CC BY 4.0).

http://creativecommons.org/licenses/by/4.0/

\section{(c) (i) Open Access}

\begin{abstract}
Background: Response inhibition and attentional flexibility are impaired in individuals with autism spectrum disorders (ASD). We descriptively analyzed whether differences in the functions of spontaneous speech derived from Antecedent-Behavior-Consequence (A-B-C) analysis are related to the T-scores for response inhibition ("Inhibit") and attentional flexibility ("Shift") of the Behavior Rating Inventory of Executive Function (BRIEF) profiles of children with ASD. Methods: Three children with ASD participated in this pilot case study. The BRIEF was used as a measure of executive function (EF). Spontaneous speech of all participants was recorded and classified into five categories: antecedent control of self (ACS), consequent control of self (CCS), antecedent control of others (ACO), consequent control of others (CCO), and exception (EXP). Results: The T-score for "Shift" of all participants exceeded a previously defined cut-off value. Spontaneous speech associated with "control of self" was not observed in any participant, while that associated with "control of others" varied among individuals. The participant with the highest frequency of spontaneous speech had the lowest "Shift" T-score, while the participant with the least active spontaneous speech had the highest "Shift" T-score. Conclusions: Our results suggest a potential association between spontaneous speech and a shift in executive function in children with ASD.
\end{abstract}




\section{Keywords}

Autism Spectrum Disorders, Spontaneous Speech, Behavior Rating Inventory of Executive Function (BRIEF), Attentional Flexibility, Antecedent-Behavior-Consequence (A-B-C) Analysis

\section{Introduction}

Children with autism spectrum disorders (ASD) exhibit deficits in social communication and restricted and repetitive behaviors (American Psychiatric Association, 2013; de Vries \& Geurts, 2015). Indeed, routines, rituals, and repetitive patterns of behavior are among the core symptoms of ASD. Mostert-Kerckhoffs et al. (2015) suggested that response inhibition and attentional flexibility were particularly impaired in individuals with ASD. They employed a visual Stroop test in 66 individuals with ASD and found that they had slower verbal responses than 56 individuals without ASD. Individuals with ASD required more reaction time to perform stop and change responses to stimuli.

Various tests can be used to evaluate executive function (EF) deficits in children with ASD (Blijd-Hoogewys, Bezemer, \& van Geert, 2014). The Behavior Rating Inventory of Executive Function (BRIEF) is a widely used behavioral rating scale measure. It assesses how a child performs in complex, unstructured everyday problem-solving situations (Blijd-Hoogewys, Bezemer, \& van Geert, 2014), indicates areas of weakness, and recommends that a clinician assess the clinical severity of the deficits (Gioia, Isquith, Guy, \& Kenworthy, 2000). In a different approach to evaluate EF, Tarbox et al. (2011) taught children with autism to respond to simple rules of "if/then" antecedent-behavior conditions, and observed a varied profile of responses. For this intervention to be successful, verbal ability must be associated with executive function (Begeer, Wierda, Scheeren, Teunisse, Koot, \& Geurts, 2014). In their verbal fluency test in 26 children and adolescents with ASD, for example, Begeer et al. (2014) used the number of words freely recalled by the participants as a measure of cognitive flexibility. However, the relationship between executive function in everyday life according to BRIEF and verbal ability has not been investigated (Semrud-Clikeman, Fine, \& Bledsoe, 2014), and it is unclear whether differences in the functions of spontaneous speech used by children with ASD are related to response inhibition and attentional flexibility.

The function of spontaneous speech can be estimated by using by Antecedent-Behavior-Consequence (A-B-C) analysis. The ability of response inhibition and attentional flexibility in children with ASD can be measured by BRIEF score. In this brief report, we aimed to descriptively analyze whether differences in the function of spontaneous speech derived by Antecedent-Behavior-Consequence (A-B-C) analysis were related to the T-scores for response inhibition and attentional flexibility, according to BRIEF, in children with ASD. 


\section{Methods}

\subsection{Participants}

One girl (Yuri, $6^{\text {th }}$ grade) and two boys (Tomo, $5^{\text {th }}$ grade and Hika, $3^{\text {rd }}$ grade) were recruited by the second author. Their teacher had referred all three students for participation because they had difficulty controlling their behavior in the classroom without prompting from adults. They were all diagnosed with ASD ( Yuri) or broader autism phenotype (Tomo and Hika) by a mental health professional (psychiatrist). In addition, the school's homeroom teacher informed us that Tomo and Hika exhibited prominent symptoms of emotional disturbance. Apart from information on her low overall activity level and slow physical movement, we had no additional information on Yuri. And there were no information of psychometric test score for them.

The procedures used here were reviewed and judged to comply with ethical standards by the Ethical Review Board of the College of Contemporary Psychology, Rikkyo University (\#17-01).

\subsection{Behavior Rating Inventory of Executive Function}

EF was assessed using the BRIEF Teacher Form (Gioia et al., 2000). We scored responses according to the BRIEF manual, and calculated the T-score for eight subcategories: Inhibit, Shift, Emotional control, Initiate, Working memory, Plan/organize, Organization of materials, and Monitor. These subcategories are clinical scales that comprise two broader indexes, the Behavioral Regulation Index (BRI) and Metacognition Index (MI) (Gioia et al., 2000). Blijd-Hoogewys et al. (2014) reported that children with ASD show high "Inhibit" and "Shift" responses in their BRI profiles. We therefore focused on the "Inhibit" and "Shift" subcategories of BRIEF in the three participants.

\subsection{Analysis of Spontaneous Speech}

We examined the influence of spontaneous speech in children with ASD on the behaviors of themselves and others. The spontaneous speech of all participants was analyzed according the A-B-C format, where " $\mathrm{A}$ " refers to the antecedent, or the event or activity that immediately precedes a behavior; " $\mathrm{B}$ " refers to the observed behavior; and "C" refers to the consequence, or the event that immediately follows a response. Observations of spontaneous speech were conducted using the event sampling method (Barlow \& Hersen, 1984). Functions of spontaneous speech (frequency per minute) were classified according to five categories: antecedent control of self (ACS), consequent control of self (CCS), antecedent control of others (ACO), consequent control of others (CCO), and exception (EXP). Individuals with ASD who exhibit high levels of spontaneous speech associated with "control of self" may show a change in response inhibition and attentional flexibility (Mostert-Kerckhoffs, Staal, Houben, \& de Jonge, 2015). 


\subsection{Procedures}

Interpersonal interactions between the participants and their classmates during school recess were recorded on video, and spontaneous speech behaviors were analyzed according to the A-B-C format. The recorded observations lasted for 5 to 22 minutes and were collected twice for each participant at an interval of one month. The first author and research colleagues (two undergraduate students) independently scored the behaviors. Interobserver agreement for the recorded spontaneous speech behaviors among the participants was $86.7 \%$ (range $75.0 \%$ - 100\%). If the frequency per minute of the control of self (CS) is high, there is a possibility of a change in response inhibition and attentional flexibility (in the BRIEF score) pointed out by individuals with ASD (Mostert-Kerckhoffs et al., 2015).

\section{Results}

The BRIEF profiles of the participants are shown in Figure 1. All participants showed a divergence with the typical profile of children with ASD reported by Blijd-Hoogewys et al. (2014). Additionally, individual differences in profiles were seen among the participants. In Yuris profile, the T-score for the "Shift" ( T-score $=77)$ and "Initiate" (T-score $=77)$ subcategories exceeded the cut-off value reported by Blijd-Hoogewys et al. (2014). In Tomo's profile, the T-score for the "Inhibit" (T-score $=69)$, "Shift" (T-score $=74)$, "Emotional control" ( -score $=81)$, "Initiate" ( $\mathrm{T}$-score $=69)$, and "Monitor" ( $\mathrm{T}$-score $=71)$ subcategories exceeded the cut-off value. In Hika's profile, the T-score for the "Inhibit" (T-score $=73$ ), "Shift" (T-score $=68)$, "Emotional control" ( $\mathrm{T}$-score $=81$ ), "Working memory" $(\mathrm{T}$-score $=74)$, "Plan/organize" $(\mathrm{T}$-score $=72)$, and "Moni-

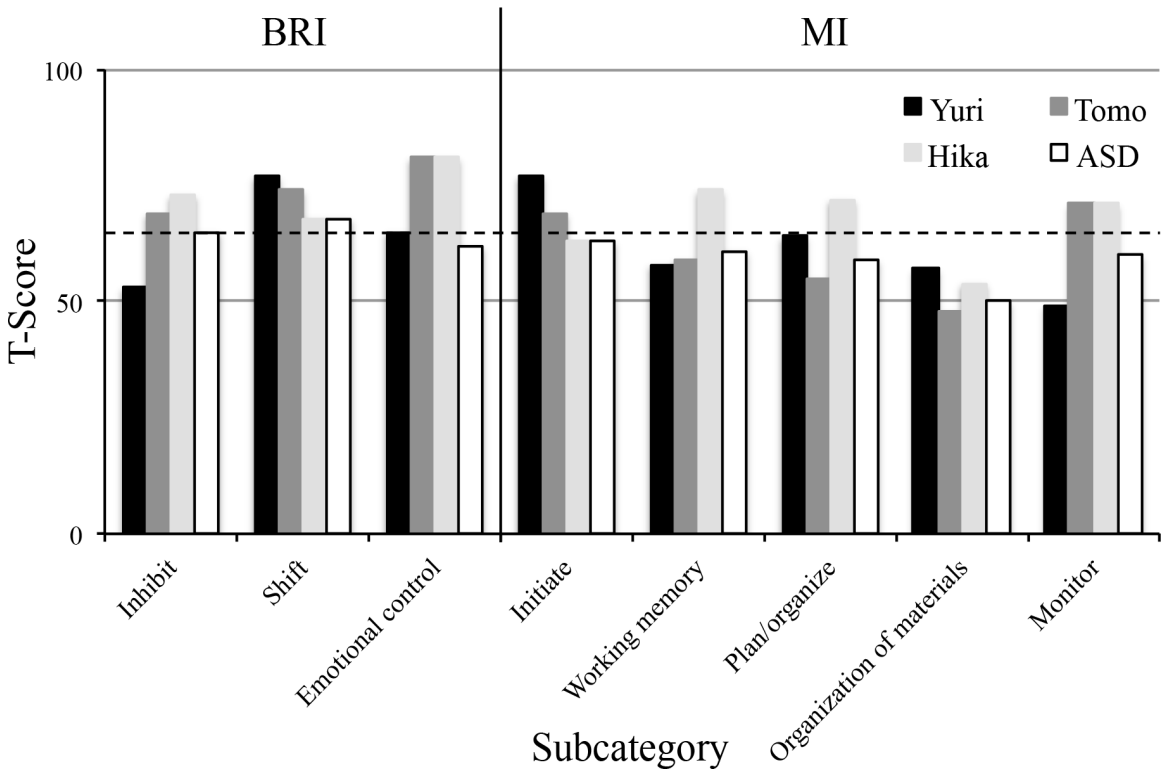

BRI = Behavioral Regulation Index; $\mathrm{MI}=$ Metacognition Index; ASD = Typical profile of children with ASD (Blijd-Hoogewys et al., 2014).

Figure 1. BRIEF profiles of the participants. Horizontal dashed line indicates the cut-off T-score at 65. 

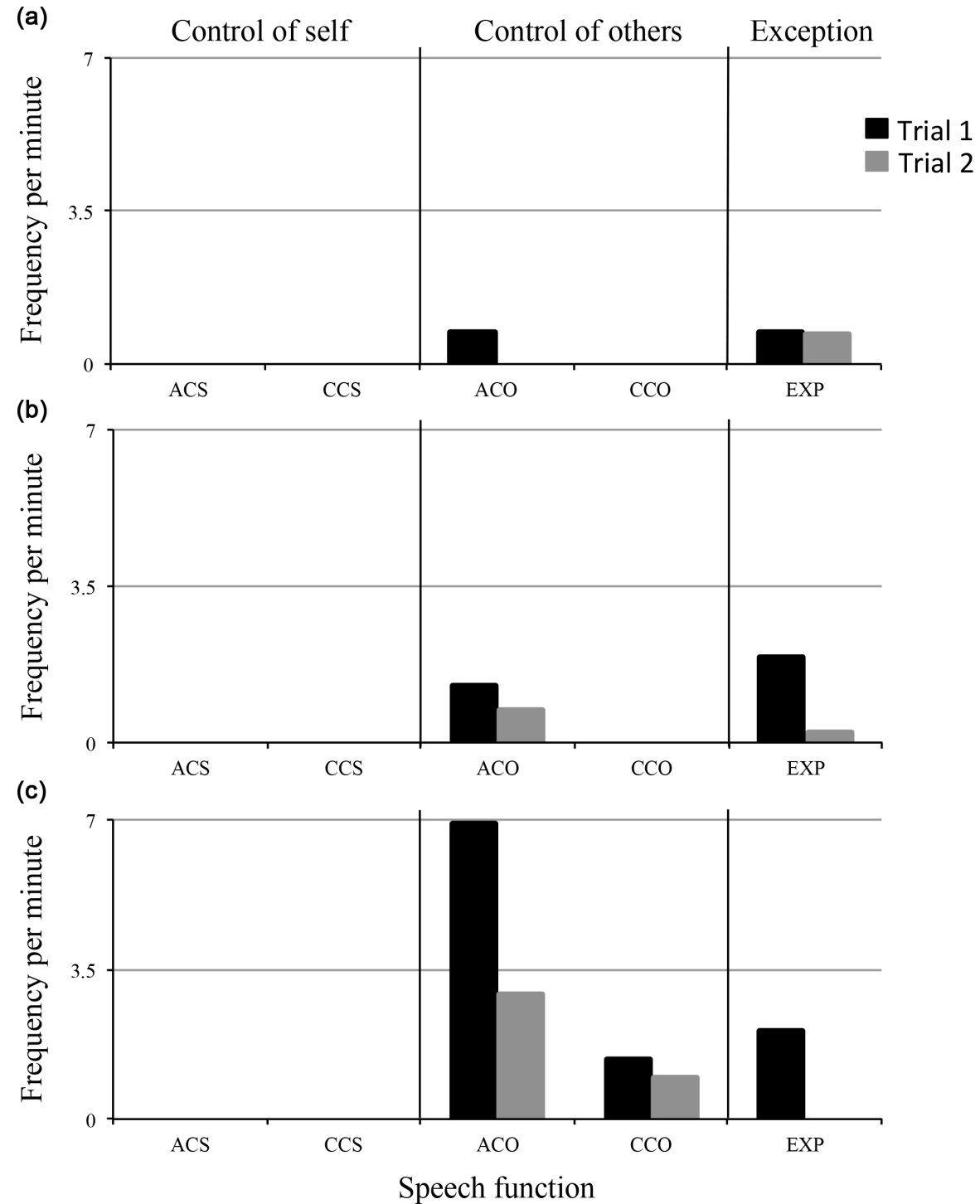

ACS $=$ Antecedent Control of Self; CCS $=$ Consequent Control of Self; ACO $=$ Antecedent Control of Others; $\mathrm{CCO}=$ Consequent Control of Others; EXP = Exception

Figure 2. Spontaneous speech functions of the participants: (a) Yuri; (b) Tomo; (c) Hika.

tor" (T-score $=71)$ subcategories exceeded the cut-off value. The T-score for "Shift" exceeded the cut-off value by a similar amount for all participants.

The spontaneous speech functions of the participants are shown in Figure 2. None of the participants exhibited spontaneous speech associated with the function of "control of self". In contrast, spontaneous speech associated with the function of "control of others" was seen in all participants, but its frequency differed markedly. Yuri showed low frequency of spontaneous speech associated with ACO (0.74/min). Tomo also only exhibited spontaneous speech associated with ACO (trial 1: 1.28/min, trial 2: $0.72 / \mathrm{min}$ ) but at a slightly higher frequency than Yuri. Hika exhibited spontaneous speech associated with both ACO (trail 1: 6.90/min, trial 2: $2.89 / \mathrm{min}$ ) and CCO (trial 1: $1.91 / \mathrm{min}$, trial 2: $0.24 / \mathrm{min}$ ) with high frequency. 
Yuri exhibited the lowest frequency of spontaneous speech but the highest "Shift" T-score among the three participants. Tomo exhibited more spontaneous speech than Yuri, but had a lower "Shift" T-score. On the other hand, Hika showed hyperactivity, exhibiting the most frequent spontaneous speech, the lowest "Shift" T-score, and highest "Inhibit" T-score among the three participants.

\section{Discussion}

Our results suggest that the spontaneous speech of children with ASD may be related to a shift in attentional flexibility among the subcategories of executive function. Our results do not suggest an association between the spontaneous speech of children with ASD and response inhibition. Spontaneous speech associated with the function of "control of self" was not observed in any of the participants. Moreover, among the "control of others" subcategories, spontaneous speech associated with CCO was not observed in 2 of the 3 participants. The participant with the highest frequency of spontaneous speech had the lowest "Shift" T-score according to BRIEF, while the participant with the least active spontaneous speech had the highest "Shift" T-score.

Our results are in agreement with previous findings. According to Begeer et al. (2014), children with ASD produce errors in clustering and switching in a verbal fluency task, suggesting that these features may be linked to cognitive flexibility. Since shifts in executive function are one of the components comprising cognitive flexibility, it is possible that the language function (verbal fluency) is related to this shift. On the other hand, our findings suggest that the "Inhibit" subcategory of BRIEF is highly associated with overall activity level, but its relationship with spontaneous speech is unclear. However, due to the limited number of cases in the present study, further studies are required to clarify these associations.

\subsection{Implications}

Our results suggest a potential association between spontaneous speech and shifts in executive function. Inducing spontaneous speech associated with a function of "control of self" may improve cognitive flexibility in children with ASD. Findings by Tarbox et al. (2011) suggest that behavioral training, by generating a verbal scenario using "if/then" conditions, can be used to improve a person's flexibility with respect to how they deal with various situations. This technique enhances self-control of behavior using rule-governed behavior (RGB) training. More research with a larger number of cases is needed to confirm these findings.

The relationship between spontaneous speech and inhibition of executive function is unclear. While an advantage of our study was that we examined participants' interpersonal interactions in the natural setting of school recess, we were unable to control for participants' activity levels. A more controlled setting may allow for more detailed analysis and confirm whether differences in settings 
affect the activities and behaviors of children with ASD. Future research should confirm behaviors in clinical and experimental settings, including those with conditionally controlled settings, activities, and behaviors.

\subsection{Limitations}

Our study has some limitations. Our results were obtained from only three cases; therefore, larger scale studies are needed to clarify the relationship between spontaneous speech and shifts in attentional flexibility. Previous studies have shown a correlation between vocabulary and similarity scores in intelligence tests (Begeer, Wierda, Scheeren, Teunisse, Koot, \& Geurts, 2014). As we only examined the relationship between spontaneous speech and executive function, further research is required to investigate the relationship between other types of speech and execution function. Moreover, we analyzed behaviors of spontaneous speech across observation sessions ranging from 5 to 22 minutes; therefore, there is a possibility that the differences observed among the participants may be attributable to differences in observation time. We do not think that this is the case, however, because the participants did not frequently exhibit a particular reaction at specific times and those who expressed behaviors at high frequency also exhibited the behavior throughout the observation period, and no differences in distribution were observed over time. To confirm this, future studies should analyze behaviors across the same length of time.

\section{Conclusion}

Our results suggest that the spontaneous speech of children with ASD may be related to a shift in attentional flexibility among the subcategories of executive function. On the other hand, our findings suggest that the "Inhibit" subcategory of BRIEF is highly associated with overall activity level, but its relationship with spontaneous speech is unclear. Our results suggest that inducing spontaneous speech associated with a function of "control of self" may improve cognitive flexibility in children with ASD.

\section{Acknowledgements}

We are grateful to the children and their families, whose participation and collaboration made this study possible.

\section{Conflicts of Interest}

The authors declare that they have no conflicts of interest.

\section{Funding}

This work was supported by JSPS KAKENHI Grant Number JP15K04253.

\section{References}

American Psychiatric Association (2013). Diagnostic and Statistical Manual of Mental 
Disorders. (5th ed.). Arlington, VA: American Psychiatric Association. https://doi.org/10.1176/appi.books.9780890425596

Barlow, D. H., \& Hersen, M. (1984). Single Case Experimental Designs: Strategies for Studying Behavior Change. London: Pergamon Press.

Begeer, S., Wierda, M., Scheeren, A. M., Teunisse, J. P., Koot, H. M., \& Geurts, H. M. (2014). Verbal Fluency in Children with Autism Spectrum Disorders: Clustering and Switching Strategies. Autism, 18, 1014-1018. https://doi.org/10.1177/1362361313500381

Blijd-Hoogewys, E. M., Bezemer, M. L., \& van Geert, P. L. (2014). Executive Functioning in Children with ASD: An Analysis of the BRIEF. Journal of Autism and Developmental Disorders, 44, 3089-3100. https://doi.org/10.1007/s10803-014-2176-9

de Vries, M., \& Geurts, H. (2015). Influence of Autism Traits and Executive Functioning on Quality of Life in Children with an Autism Spectrum Disorder. Journal of Autism and Developmental Disorders, 45, 2734-2743.

https://doi.org/10.1007/s10803-015-2438-1

Gioia, G. A., Isquith, P. K., Guy, S. C., \& Kenworthy, L. (2000). Behavior Rating Inventory of Executive Function. Luts, Florida: PAR.

Mostert-Kerckhoffs, M. A., Staal, W. G., Houben, R. H., \& de Jonge, M. V. (2015). Stop and Change: Inhibition and Flexibility Skills Are Related to Repetitive Behavior in Children and Young Adults with Autism Spectrum Disorders. Journal of Autism and Developmental Disorders, 45, 3148-3158. https://doi.org/10.1007/s10803-015-2473-y

Semrud-Clikeman, M., Fine, J. G., \& Bledsoe, J. (2014). Comparison among Children with Autism Spectrum Disorder, Nonverbal Learning Disorder and Typically Developing Children on Measures of Executive Functioning. Journal of Autism and Developmental Disorders, 44, 331-342. https://doi.org/10.1007/s10803-013-1871-2

Tarbox, J., Zuckerman, C. K., Bishop, M. R., Olive, M. L., \& O’Hora, D. P. (2011). Rule-Governed Behavior: Teaching a Preliminary Repertoire of Rule-Following to Children with Autism. The Analysis of Verbal Behavior, 27, 125-139.

https://doi.org/10.1007/BF03393096 